\title{
AClR
}

Selected Papers of \#AolR2019:

The $20^{\text {th }}$ Annual Conference of the Association of Internet Researchers Brisbane, Australia / 2-5 October 2019

\section{MAKING SENSE OF SOCIAL MEDIA PRIVACY: A FRAMING EXPERIMENT}

\author{
Dmitry Epstein \\ Hebrew University of Jerusalem \\ Kelly Quinn \\ University of Illinois at Chicago
}

Given the conceptual fluidity of privacy, its framing in both popular and policy discourses is increasingly important (Epstein, Roth, \& Baumer, 2014; Mukherjee, 2000), especially as trading of privacy for information and services has become commonplace. Framing offers individuals "schemata of interpretation" (Goffman, 1974, p. 21) or "a central organizing idea" (Gamson \& Modigliani, 1989, p. 3) for sense-making of complex, highly contextualized issues. In policy discourse, frames define problems, set priorities, and suggest solutions; media frames, are likewise influential, as they establish a common ground linking elite and non-elite discourses, and have the potential to sway public opinion (Chong \& Druckman, 2007). While the mechanisms of framing have been excessively studied in a variety of politically-charged contexts such as nuclear power (Gamson \& Modigliani, 1989) and climate change (Nisbet, Hart, Myers, \& Ellithorpe, 2013), there is limited research on the framing of privacy (Epstein, Roth, \& Baumer, 2014). This paper aims to help addressing this lacuna.

Literature on framing distinguishes between frames in thought and frames in communication. The former refer to the considerations and associated subjective values that impact an individual's evaluation of a given problem or a situation, thus demarcating the range of possible or desirable alternatives. The latter, also sometimes referred to as media frames, relate to the words and styles that an elite speaker typically uses, either intentionally or unintentionally, when relaying information about an issue to an audience; these typically align with the values and priorities of the involved parties (Chong \& Druckman, 2007). 
Research on the framing of privacy has suggested that while elites tend to use vertical (or institutional) privacy frames in communication (Epstein, et al., 2014), non-elites tend to hold onto horizontal (or social) privacy frames in thought (Quinn \& Epstein, 2018). The question, thus, is what happens to the individual's privacy concerns and attitudes when their frames in thought about privacy collide with the frames in communication they are exposed to from authoritative sources. Specifically, we ask:

RQ1: What is the relationship between an individual's 'frames in thought' about privacy (vertical/horizontal) and their privacy concerns and privacy self-efficacy?

RQ2: How does vertical/horizontal privacy framing in communication affect an individual's privacy concerns and privacy self-efficacy?

RQ3: What effect does (lack of) consistency between one's 'frames in thought' and 'frames in communication' have on privacy concerns and privacy selfefficacy?

\section{Method}

We explore the relationship between frames in communication, frames in thought, and associated privacy concerns and self efficacy from three different perspectives. First, we examine whether an individual's 'frame in thought', as either vertically- or horizontallyoriented, results in differing levels of privacy concern and privacy self-efficacy. Next, we probe whether introducing either a horizontal or vertical 'frame in communication' alters these same outcomes. Finally, we explore whether there is a difference in privacy concerns or privacy self-efficacy when the 'frame in communication' is consistent, or alternatively inconsistent, with the participant's 'frame in thought.'

We draw on survey-based experiment conducted with a unique sample of US social media users. Participants $(n=628)$ were recruited in June-August, 2018 through a Qualtrics panel service and surveyed on their social media privacy attitudes and behaviors. Embedded in the survey was a $2 \times 2$ experiment which first ascertained a participant's privacy orientations as horizontal or vertical, and then randomly assigned participants into a vertical or horizontal 'frames in communication' condition (i.e. participants 'frame in thought' was either reinforced or contradicted with a 'frame in communication'). Following this manipulation, participants were asked a series of questions about their social media privacy concerns and privacy self-efficacy.

Privacy concerns were measured with both horizontal and vertical dimensions: horizontal concerns centered on the use of one's personal information by other individuals who used the social media platform; and vertical concerns focused on the use of user-supplied information by platform sponsors and other institutional actors 
(such as government agencies). Privacy self-efficacy assessed the degree to which the participant felt in control of who could view their social media information and confidence in what information could be shared by others.

\section{Results}

We conducted a series of ANOVA analyses to examine these framing effects. Addressing RQ1, which explored the effects of frames in thought, we found no significant difference in privacy concerns (horizontal $F_{1,626}=.14, p=.71$; vertical

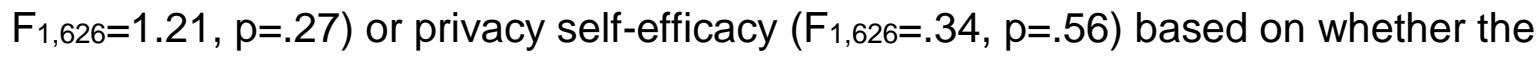
individual frames privacy in horizontal or vertical terms. RQ2 considered frames in communication, and whether a horizontally- or vertically-oriented message resulted in differing levels of privacy concerns. Here, we found an indication that receiving a message about privacy that is horizontally/socially focused results in higher levels of privacy self-efficacy $\left(F_{1,588.87}=10.24, p=.001\right)$, but has no effect on either horizontal $\left(F_{1,626}=2.04, p=.15\right)$ or vertical $\left(F_{1,626=1.31, p}=.25\right)$ privacy concerns. Finally, RQ3 explored whether a message consistent with the participant's frame in thought about privacy resulted in a different level of privacy concern. Here, we found that messaging consistent with the participant's frame in thought resulted in lower levels of privacy concerns overall, with vertically-oriented privacy concerns $\left(F_{1,626}=3.85, p=.05\right)$ demonstrating significant differences. There were no significant differences in privacy self-efficacy between those receiving either a consistent and inconsistent frame in communication.

\section{Implications}

With privacy taking central stage in the contemporary information society, it is important to pay attention to how this complex issue is communicated to the public. Potential implications of this study can be viewed on both strategic and tactical levels. On the strategic level, our findings reinforce the idea that the framing of privacy matters. As such, privacy framing mechanisms deserve additional scrutiny by scholars and additional efforts by activists to make said mechanisms explicit. On the tactical level we observe how communicating about privacy in horizontal terms - a strategy often times employed by social media platform providers - makes people more confident in their ability to protect their privacy, despite whether this is actually the case. Such dynamics may play into preservation of existing power structures where individuals provide personal information without considering its institutional uses. If we subscribe to the idea that critical participation in the information society is beneficial overall, findings about the (in)consistency of privacy framing highlight the importance of both research and education to the multidimensionality of privacy as a pivotal concept. 
Our findings may of course reflect the limitations associated with online panel survey sampling, self-reported data, and known weakness associated with experimental studies, but it offers insights that both the policy and scholarly community should be grappling with. Our further work will delve deeper into unpacking the relationships between framing, confounding factors, and the relationships between them. First, we will highlight relationships between demographic characteristics and 'frames in thought,' to explore structural influences on how privacy is conceptualized. Second, we will explore in greater detail how 'frames in thought' as vertically- or horizontally-oriented are differentially impacted by (in)consistency in 'frames in communication.'

\section{References}

Chong, D., \& Druckman, J. N. (2007). Framing theory. Annual Review of Political Science, 10, 103-126.

Epstein, D., Roth, M. C., \& Baumer, E. P. S. (2014). It's the Definition Stupid! Framing of Online Privacy in the Internet Governance Forum Debates. Journal of Information Policy, 4, 144-172.

Gamson, W. A., \& Modigliani, A. (1989). Media discourse and public opinion on nuclear power: A constructionist approach. American Journal of Sociology, 95(1), 1.

Goffman, E. (1974). Frame analysis. Cambridge, MA: Harvard University Press.

Mukherjee, R. (2000). "Now you see it, now you don't": Naming privacy, framing policy. Critical Studies in Media Communication, 17(4), 469-492. https://doi.org/10.1080/15295030009388414

Nisbet, E. C., Hart, P. S., Myers, T., \& Ellithorpe, M. (2013). Attitude Change in Competitive Framing Environments? Open-/Closed-Mindedness, Framing Effects, and Climate Change. Journal of Communication, 63(4), 766-785. https://doi.org/10.1111/jcom.12040

Quinn, K., \& Epstein, D. (2018). \#MyPrivacy: How users think about social media privacy. In Proceedings of the 9th International Conference on Social Media and Society (pp. 360-364). New York, NY, USA: ACM.

https://doi.org/10.1145/3217804.3217945 